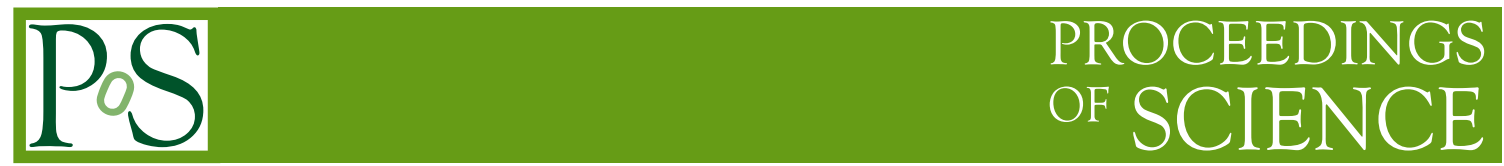

\title{
Radiative Kaon decays: where do we stand?
}

\section{Oscar Catà}

Theoretische Physik 1, Universität Siegen, Walter-Flex-Straße 3, D-57068 Siegen, Germany

E-mail: oscar.cataduni-siegen.de

Radiative kaon decays are extremely relevant modes in the search for physics beyond the Standard Model, as well as to learn about the Standard Model itself below the confinement scale. The field experienced a vigorous boost due to the scientific programs started by $\mathrm{KTeV}$ and NA48 more than 20 years ago. In this write-up I will focus on the potential of the radiative kaon analyses to be performed at NA62 and LHCb to constrain further the Standard Model at low energies.

XIV International Conference on Heavy Quarks and Leptons (HQL2018)

May 27- June 1, 2018

Yamagata Terrsa, Yamagata,Japan 


\section{Introduction}

Rare kaon decays are a very fertile field of study, well suited for searches of new physics but also able to provide very relevant tests of the Standard Model below the GeV scale. Besides the important experimental activity of the past, the currently ongoing experimental facilities devoted to kaon physics ensure that the field will be invigorated with new data: KOTO will test $K_{L} \rightarrow \pi^{0} \bar{v} v$ for the first time, NA62 is entirely devoted to kaon physics, and LHCb has a very ambitious program on rare $K_{S}$ decays.

In this manuscript I will focus on the subset of radiative kaon decays of the form $K \rightarrow \pi \gamma^{(*)}$ and $K \rightarrow \pi \pi \gamma^{(*)}$, either with real photons or lepton pairs, paying special attention to those modes that NA62 and LHCb will measure in the near future.

Decay modes involving real or virtual photons are dominated by long distances, with an overwhelmingly large Bremsstrahlung contribution. The importance of these long-distance dominated decay modes is twofold. First, and contrary to what one would naively expect, the long-distance dominance of these decays does not preclude them from being sensitive to new physics. The rich kinematics of the decays, especially the decays with lepton pairs, ensure a large number of differential observables. Among them, angular distributions can provide rather clean new-physics tests, not restricted to CP-violating observables only [1]. Second, they are important to constraint the $\Delta S=1$ sector of the Standard Model at low energies. At the kaon scale, quarks are confined and the Standard Model takes the form of Chiral Perturbation Theory, where the coefficients of the theory (the weak chiral couplings) cannot be computed from first principles and one has to resort to hadronic models. Experimental input is therefore essential to (i) get a precise determination of the chiral coefficients; and (ii) conclude whether they are compatible with models. However, in order to isolate the chiral coefficients, a percent precision is typically needed, which makes their experimental determination quite a challenging task.

In this note I want to bring attention to an interesting fact: the experimental situation in radiative kaon decays is at a point where the relevant weak chiral couplings can be individually determined and even overconstrained. In the next pages I will report on how to optimize the experimental extraction of such couplings in the decay modes $K^{+} \rightarrow \pi^{+} \pi^{0} e^{+} e^{-}$and $K_{S} \rightarrow \pi^{+} \pi^{-} e^{+} e^{-}$, to be analysed at NA48/2 and LHCb, respectively.

The upcoming data on radiative kaon decays also motivates a reexamination of the theoretical predictions given by hadronic models. This I will address at the end, and show that the agreement between experiment and theory is, within the limitations inherent to hadronic models, rather satisfactory.

\section{Status and prospects for radiative kaon decays}

The experimental studies of radiative decays containing pions were pioneered by $\mathrm{KTeV}$ and NA48, which covered a big number of these modes, including

$$
\begin{array}{lll}
K^{ \pm} \rightarrow \pi^{ \pm} \pi^{0} \gamma, & K_{S} \rightarrow \pi^{0} \gamma^{*}, & K_{S} \rightarrow \pi^{+} \pi^{-} e^{+} e^{-}, \\
K^{ \pm} \rightarrow \pi^{ \pm} \gamma \gamma, & K_{S} \rightarrow \pi^{0} \gamma \gamma, & K_{L} \rightarrow \pi^{+} \pi^{-} e^{+} e^{-}, \\
K^{ \pm} \rightarrow \pi^{ \pm} \gamma^{*} & &
\end{array}
$$




\begin{tabular}{c|c|c} 
Decay mode & counterterm combination & expt. value \\
\hline$K^{ \pm} \rightarrow \pi^{ \pm} \gamma^{*}$ & $N_{14}-N_{15}$ & $-0.0167(13)$ \\
\hline$K_{S} \rightarrow \pi^{0} \gamma^{*}$ & $2 N_{14}+N_{15}$ & $+0.016(4)$ \\
\hline$K^{ \pm} \rightarrow \pi^{ \pm} \pi^{0} \gamma$ & $N_{14}-N_{15}-N_{16}-N_{17}$ & $+0.0022(7)$ \\
\hline$K^{ \pm} \rightarrow \pi^{ \pm} \gamma \gamma$ & $N_{14}-N_{15}-2 N_{18}$ & $-0.0017(32)$
\end{tabular}

Table 1: Values of the weak couplings together with the decay mode from which they can most precisely be extracted at present.

Besides measuring the decay rate, information on weak chiral couplings was extracted for some of the decay modes. The strategy used to overcome the Bremsstrahlung dominance was to study the interference term between the Bremsstrahlung and resonance contributions, which is substantially more sizeable than the pure resonance piece squared. Since the interference term is linear in the weak chiral couplings, this has the additional advantage that one is sensitive not just to their magnitude, but also to their sign. Numbers can be obtained by measuring the slopes of the differential decay rates in terms of final-state particle energies. The most updated results are [2]

$$
\begin{array}{ll}
K^{ \pm} \rightarrow \pi^{ \pm} \gamma^{*}: & a_{+}=-0.578 \pm 0.016 \\
K_{S} \rightarrow \pi^{0} \gamma^{*}: & a_{S}=\left(1.06_{-0.21}^{+0.26} \pm 0.07\right) \\
K^{ \pm} \rightarrow \pi^{ \pm} \pi^{0} \gamma: & X_{E}=(-24 \pm 4 \pm 4) \mathrm{GeV}^{-4} \\
K^{+} \rightarrow \pi^{+} \gamma \gamma: & \hat{c}=1.56 \pm 0.23 \pm 0.11,
\end{array}
$$

which are linked to the chiral couplings as [3]

$$
\begin{aligned}
\mathscr{N}_{E}^{(1)} & \equiv N_{14}-N_{15}=\frac{3}{64 \pi^{2}}\left(\frac{1}{3}-\frac{G_{F}}{G_{8}} a_{+}-\frac{1}{3} \log \frac{\mu^{2}}{m_{K} m_{\pi}}\right)-3 L_{9}, \\
\mathscr{N}_{S} & \equiv 2 N_{14}+N_{15}=\frac{3}{32 \pi^{2}}\left(\frac{1}{3}+\frac{G_{F}}{G_{8}} a_{S}-\frac{1}{3} \log \frac{\mu^{2}}{m_{K}^{2}}\right), \\
\mathscr{N}_{E}^{(0)} & \equiv N_{14}-N_{15}-N_{16}-N_{17}=-\frac{\left|\mathscr{M}_{K}\right| f_{\pi}}{2 G_{8}} X_{E}, \\
\mathscr{N}_{0} & \equiv N_{14}-N_{15}-2 N_{18}=\frac{3}{128 \pi^{2}} \hat{c}-3\left(L_{9}+L_{10}\right) .
\end{aligned}
$$

The numerical values of the different combinations are listed in Table 1.

As to the near-future prospects, NA48/2 has data on $K^{+} \rightarrow \pi^{+} \pi^{0} e^{+} e^{-}$, stored but not yet fully analysed [4]. As I will discuss in the next section, this is a very interesting decay mode. From Table 1 it is clear that while $N_{14}, N_{15}$ and $N_{18}$ can be determined, only $N_{16}+N_{17}$ has been probed. $K^{+} \rightarrow \pi^{+} \pi^{0} e^{+} e^{-}$has an orthogonal combination that, if measured, would allow a separate determination of $N_{16}$ and $N_{17}$. NA62 will in principle also collect data for this mode, though at the moment it is optimized to measure $K^{+} \rightarrow \pi^{+} \bar{v} v$.

In turn, LHCb plans to examine the mode $K_{S} \rightarrow \pi^{+} \pi^{-} e^{+} e^{-}$[5], for which so far only the branching ratio is known. As I will discuss below, if enough precision is reached, this mode can be used to improve the determination of $N_{14}, N_{15}$ and $N_{16}+N_{17}$, but it does not provide an independent weak chiral combination to disentangle $N_{16}$ and $N_{17}$, as $K^{+} \rightarrow \pi^{+} \pi^{0} e^{+} e^{-}$does. 
Other decay modes accessible at LHCb will be $K_{S} \rightarrow \mu^{+} \mu^{-}, K_{S} \rightarrow \gamma \mu^{+} \mu^{-}$or $K_{S} \rightarrow \mu^{+} \mu^{-} \mu^{+} \mu^{-}$. I will not discuss them in this review. These modes are however very interesting and theoretically interrelated (see [6] for a discussion).

\section{Theoretical aspects of radiative 4-body decays}

Radiative 4-body decays are very interesting rare decay modes. They are dominated by longdistance physics yet their rich kinematics allow one to study a number of distributions. Particularly interesting are the angular distributions, with which one can test new physics beyond CP-violating observables (see [1]), and the distribution in the lepton pair invariant mass. The latter is especially important for chiral tests.

Kaon decays are described by the $\Delta S=1$ sector of chiral dynamics, the low-energy effective field theory of the strong interactions. The expansion up to next-to-leading order is

$$
\mathscr{L}_{\Delta S=1}=G_{8} f_{\pi}^{4} \operatorname{tr}\left[\lambda_{6} D_{\mu} U^{\dagger} D^{\mu} U\right]+G_{8} f_{\pi}^{2} \sum_{j} N_{j} W_{j}\left(U, D_{\mu} U, \lambda_{6}\right)+\mathscr{O}\left(p^{6}\right)
$$

with

$$
U=\exp \left[i \frac{\phi^{a} \tau^{a}}{f_{\pi}}\right]
$$

the chiral field and $\lambda_{6}$ the $\Delta S=1$ spurion. The leading-order piece contains one operator, determined by the coupling $G_{8} f_{\pi}^{4}$, while the 37 coefficients $N_{j}$ of the subleading term depend on hadronic physics. For the decay modes of the form $K \rightarrow \pi \gamma^{(*)}, \pi \pi \gamma^{(*)}$, only combinations of $N_{14}, \ldots, N_{18}$ and $N_{28}, \ldots, N_{31}$ are relevant.

A generic amplitude for such decay modes is of the form:

$$
\mathscr{M}\left(K \rightarrow X \gamma^{(*)}\right)=\underbrace{\mathscr{M}_{B}\left(\mathscr{O}\left(p^{2}\right)\right)}_{\text {Brems. }}+\underbrace{\mathscr{M}_{E}\left(\mathscr{O}\left(p^{4}\right)\right)}_{\text {electric, } \mathrm{CP}-\text { even }}+\underbrace{\mathscr{M}_{M}\left(\mathscr{O}\left(p^{4}\right)\right)}_{\text {magnetic, } \mathrm{CP}-\text { odd }},
$$

where 'electric' and 'magnetic' refer simply to the intrinsic parity (even parity or odd parity) of the hadronic contributions.

The Bremsstrahlung contribution is clearly the dominant one, but since it is electromagnetic, it can be related to the corresponding non-radiative decay modes through Low's theorem. The hadronic contribution is subdominant, but with the statistics expected at LHCb and NA62, the determination of the electric weak chiral couplings from $K \rightarrow \pi \pi e^{+} e^{-}$decays is likely to be achieved for the first time. So far only the magnetic ones in $K_{L} \rightarrow \pi^{+} \pi^{-} e^{+} e^{-}$have been measured. As mentioned above, in order to have an individual determination for the weak chiral couplings, besides the combinations entering the radiative 3-body decays, one extra independent combination is needed. This can only come from 4-body decays.

The strategy to extract the weak chiral couplings is similar to the one with 3-body decays. Given the size of the Bremsstrahlung contribution, one should focus on the interference term between the electric and Bremsstrahlung pieces. The extra advantage of the 4-body decays is that one can play with the dilepton invariant mass squared, $q^{2}$, to find an optimal window for the parameter extraction. This is useful because the Bremsstrahlung contribution is peaked at low values of $q^{2} \sim 4 m_{e}^{2}$. However, since for large $q^{2}$ values close to the endpoint statistics are poor, moderate 


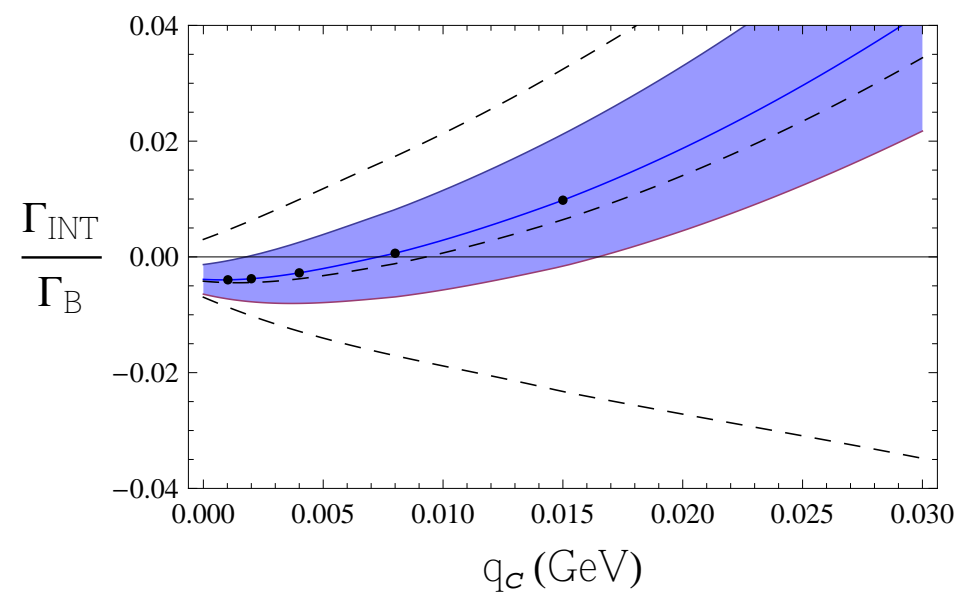

Figure 1: Dependence of the interference term on the values of the chiral couplings for $K^{+} \rightarrow \pi^{+} \pi^{0} e^{+} e^{-}$ in terms of cuts in $q^{2}$ [8]. The blue band corresponds to the experimental values for $\mathscr{N}_{E}^{(0)}, \mathscr{N}_{E}^{(1)}$ and $\mathscr{N}_{E}^{(2)}$ (with $N_{17}=0$ ). The dashed lines (from top to bottom) correspond, respectively, to setting $\mathscr{N}_{E}^{(0)}, \mathscr{N}_{E}^{(1)}$ and $\mathscr{N}_{E}^{(2)}$ to zero one at a time.

values of $q^{2}$ are eventually the ones to be preferred. This generic strategy can be applied to the different $K \rightarrow \pi \pi e^{+} e^{-}$modes. At present, a full-fledged theoretical analysis along these lines is available only for $K^{+} \rightarrow \pi^{+} \pi^{0} e^{+} e^{-}[7,1,8]$.

3.1 $K^{+} \rightarrow \pi^{+} \pi^{0} e^{+} e^{-}$

NA48/2 has collected 5000 events of this decay, which are currently being analysed [4]. A determination of the decay rate and the magnetic piece with a reasonable accuracy will be possible, and an extraction of the weak chiral couplings will be attempted.

This decay mode is sensitive to the following combinations of weak chiral couplings:

$$
\begin{aligned}
& \mathscr{N}_{E}^{(0)} \equiv N_{14}-N_{15}-N_{16}-N_{17}=+0.0022(7), \\
& \mathscr{N}_{E}^{(1)} \equiv N_{14}-N_{15}=-0.0167(13), \\
& \mathscr{N}_{E}^{(2)}=N_{14}+2 N_{15}-3\left(N_{16}-N_{17}\right),
\end{aligned}
$$

where the last one is exclusive of this decay. Using the experimental information of Table 1, it can be reexpressed as

$$
\mathscr{N}_{E}^{(2)}=+0.089(11)+6 N_{17} \text {. }
$$

The previous numerical results are entirely based on experimental input. An experimental determination of $\mathscr{N}_{E}^{(2)}$ therefore amounts to a determination of $N_{17}$. Eq. (3.5) strongly suggests that $\mathscr{N}_{E}^{(2)}$ is sizeable, almost two orders of magnitude bigger than $\mathscr{N}_{E}^{(0)}$. Only a sizeable and negative $N_{17}$ could overturn this conclusion. However, all model predictions indicate that $N_{17}$ is very small, if not vanishing.

The main problem for an experimental extraction of $\mathscr{N}_{E}^{(2)}$ is that, for the total integrated rates, $\Gamma_{\text {int }} \sim 10^{-2} \Gamma_{\text {Brems. }}$. In order to overcome the dominance of the Bremsstrahlung, the best strategy is 
to avoid low- $q^{2}$ data, where the Bremsstrahlung distribution attains its maximum. A cut at $q \sim 20$ $\mathrm{MeV}$ is typically enough to gain an order of magnitude [8].

Another important piece of information is the shape of the interference term as a function of $\mathscr{N}_{E}^{(i)}$. Fig. 1 shows that the interference term flips sign in a way controlled mostly by $\mathscr{N}_{E}^{(0)}$ and $\mathscr{N}_{E}^{(2)}$, while $\mathscr{N}_{E}^{(1)}$ plays a minor role. This pattern is of importance for the experimental extraction of $\mathscr{N}_{E}^{(2)}$.

3.2 $K_{S} \rightarrow \pi^{+} \pi^{-} e^{+} e^{-}$

In order to probe the chiral $\mathscr{O}\left(p^{4}\right)$ structure of this decay one needs to reach the percent precision, which is challenging but might be possible at $\mathrm{LHCb}$ [5].

The decay depends on $\mathscr{N}_{E}^{(0)}$ and a new combination

$$
\mathscr{N}_{E}^{(3)}=N_{14}-N_{15}-3\left(N_{16}+N_{17}\right)
$$

which can be predicted from the entries of Table 1 to be

$$
\mathscr{N}_{E}^{(3)}=-2 \mathscr{N}_{E}^{(1)}+3 \mathscr{N}_{E}^{(0)}=+0.040(5) .
$$

A particularly interesting aspect of this mode is that the magnetic piece turns out to be a CPviolating effect. This means that the electric interference term is, despite being suppressed, the relevant contribution after the Bremsstrahlung. With the values for $\mathscr{N}_{E}^{(0)}$ and $\mathscr{N}_{E}^{(3)}$, one predicts [8]

$$
B R\left(K_{S} \rightarrow \pi^{+} \pi^{-} e^{+} e^{-}\right)=\underbrace{4.74 \cdot 10^{-5}}_{\text {Brems }}+\underbrace{4.39 \cdot 10^{-8}}_{\text {int }}+\underbrace{1.33 \cdot 10^{-10}}_{\text {electric }},
$$

which agrees extremely well with the branching ratio measured by NA48 [9]:

$$
B R\left(K_{S} \rightarrow \pi^{+} \pi^{-} e^{+} e^{-}\right)_{\exp }=(4.79 \pm 0.15) \times 10^{-5} .
$$

The extraction of $\mathscr{N}_{E}^{(0)}$ and $\mathscr{N}_{E}^{(3)}$ from this decay mode is an important test to increase the precision on $N_{14}, N_{15}$ and $N_{16}+N_{17}$. The analogous decay with muons is not a viable alternative, since it is extremely suppressed by phase space: $B R\left(K_{S} \rightarrow \pi^{+} \pi^{-} \mu^{+} \mu^{-}\right) \sim 4.7 \cdot 10^{-14}[8]$.

3.3 $K_{L} \rightarrow \pi^{+} \pi^{-} e^{+} e^{-}$

It is worth mentioning that, while $K_{S} \rightarrow \pi^{+} \pi^{-} e^{+} e^{-}$does not provide information for an independent determination of $N_{16}$ and $N_{17}$, a combined analysis with $K_{L} \rightarrow \pi^{+} \pi^{-} e^{+} e^{-}$would. The branching ratio and the magnetic piece have already been measured for this decay mode [10], with results in agreement with the theoretical predictions [11, 7]. A determination of the electric interference piece would be very nice, though it is not clear whether the statistics collected by NA48 are enough for an extraction of the chiral couplings.

The weak chiral couplings entering $K_{L, S} \rightarrow \pi^{+} \pi^{-} e^{+} e^{-}$are $\mathscr{N}_{E}^{(0)}$ and

$$
\begin{aligned}
& \mathscr{N}_{E}^{(3)}=N_{14}-N_{15}-3\left(N_{16}+N_{17}\right), \\
& N_{E}^{(4)}=N_{14}-N_{15}-3\left(N_{16}-N_{17}\right) .
\end{aligned}
$$


In $K_{S} \rightarrow \pi^{+} \pi^{-} e^{+} e^{-}, \mathscr{N}_{E}^{(3)}$ dominates and $\mathscr{N}_{E}^{(4)}$ is a CP-violating contribution. In $K_{L} \rightarrow \pi^{+} \pi^{-} e^{+} e^{-}$ the roles get reversed. Therefore, an extraction of $N_{17}$ from neutral kaon decays would be possible with information on $K_{L, S} \rightarrow \pi^{+} \pi^{-} e^{+} e^{-}$, since

$$
\mathscr{N}_{E}^{(4)}-\mathscr{N}_{E}^{(3)}=6 N_{17}
$$

\section{Theoretical status}

The weak chiral couplings are unspecified parameters of the chiral Lagrangian. From a theoretical viewpoint, their determination demands methods that go beyond chiral symmetry.

In the pure strong sector, the experimental values of the NLO coefficients fit reasonably well the expectations of lowest-meson dominance. In the $\Delta S=1$ the situation is notoriously more difficult, not just because the number of operators is substantially larger, but because the couplings are sensitive to the whole range of energies. Resonance-based models [12] thus rest on the assumption that the low-energy region dominates. On top of this assumption, given the large number of parameters to fit, additional simplifying assumptions are used in order to end up with predictive schemes. This means that discrepancies with experimental determinations can be caused by (a) non-negligible short-distance contributions and/or (b) assumptions on the long-distance contributions which are not supported phenomenologically.

For these reasons, caution has to be exercised when contrasting experimental data to models. However, it is reassuring that the following qualitative prediction pattern for the weak chiral couplings is quite generic:

$$
\begin{aligned}
& N_{14}-N_{15} \simeq+\mathscr{O}\left(10^{-2}\right) ; \quad N_{14}+2 N_{15}-3\left(N_{16}-N_{17}\right) \simeq+\mathscr{O}\left(10^{-2}\right) ; \\
& 2 N_{14}+N_{15} \simeq-\mathscr{O}\left(10^{-2}\right) ; \quad N_{14}-N_{15}-3\left(N_{16}-N_{17}\right) \simeq+\mathscr{O}\left(10^{-2}\right) ; \\
& N_{14}-N_{15}-N_{16}-N_{17} \simeq \mathscr{O}\left(10^{-3}\right) ; \quad N_{14}-N_{15}-3\left(N_{16}+N_{17}\right) \simeq+\mathscr{O}\left(10^{-2}\right) ; \\
& N_{14}-N_{15}-2 N_{18} \simeq \mathscr{O}\left(10^{-3}\right) ; \quad 7\left(N_{14}-N_{16}\right)+5\left(N_{15}+N_{17}\right) \simeq+\mathscr{O}\left(10^{-1}\right) .
\end{aligned}
$$

The left column corresponds to already measured quantitites (see Table 1) and agrees with experimental numbers. The right column is still at the level of untested predictions. It is interesting to notice the spread of numbers between $\mathscr{O}\left(10^{-1}\right)$ and $\mathscr{O}\left(10^{-3}\right)$. Models ascribe the $\mathscr{O}\left(10^{-3}\right)$ values to accidental cancellations, proportional to $\left(L_{9}+L_{10}\right)$, which is known to be a small number. It can therefore not be called a discrepancy that, e.g., the theoretical and experimental values of $N_{14}-N_{15}-N_{16}-N_{17}$ turned out to have opposite signs, given that the expected precision of the models cannot go beyond $\mathscr{O}\left(10^{-3}\right)$.

\section{Conclusions}

The experimental situation in radiative kaon decays is at a point where the relevant (electric) weak chiral counterterms $N_{14}$ to $N_{18}$ can be individually determined. From $K \rightarrow \pi \gamma^{*}$ decays, one finds

$$
N_{14}=(-2 \pm 18) \times 10^{-4} ; \quad N_{15}=(1.65 \pm 0.22) \times 10^{-2}
$$


and adding $K \rightarrow \pi \gamma \gamma$, one gets

$$
N_{18}=(-7.5 \pm 2.3) \times 10^{-3}
$$

With 3-body radiative decays, only the combination $N_{16}+N_{17}$ can be measured. In order to go further, 4-body decays are needed. These decay modes are not only important to determine with precision the Standard Model at low energies, but they have a rich kinematical structure that makes them also interesting probes of new physics. The analyses of the decay modes $K^{ \pm} \rightarrow \pi^{ \pm} \pi^{0} e^{+} e^{-}$ at NA48 and $K_{S} \rightarrow \pi^{+} \pi^{-} e^{+} e^{-}$at LHCb are an important step in this direction. In particular, the former has the potential to provide for the first time a determination of $N_{16}$ and $N_{17}$. Strategies to compensate the overwhelming dominance of the Bremsstrahlung contribution are well-developed and can be useful, especially taken into account that statistics might be rather limited.

LHCb and NA62 will continue their data taking on radiative kaon decays and analyze decay modes other than the ones mentioned above. In this context, it would be very interesting to access the $\mathscr{O}\left(p^{4}\right)$ chiral couplings through $K_{L} \rightarrow \pi^{+} \pi^{-} e^{+} e^{-}$which, in combination with $K_{S} \rightarrow$ $\pi^{+} \pi^{-} e^{+} e^{-}$, can provide very interesting information.

Note added: After the completion of these Proceedings, the first detection and analysis of $K^{+} \rightarrow \pi^{+} \pi^{0} e^{+} e^{-}$by NA48/2 was made public [13]. With a bit less than 5000 events, they determined the decay rate to a $3 \%$ precision. Their number, $B R\left(K^{+} \rightarrow \pi^{+} \pi^{0} e^{+} e^{-}\right) \exp =(4.24 \pm 0.14) \times$ $10^{-6}$, is in excellent agreement with the theoretical prediction [1]. Both the magnetic contribution (a percent effect) and the electric interference term (a permile effect) were extracted through a Dalitz plot analysis. In both cases the results were in agreement with the long-distance estimates made in [1] and [8], respectively. Higher statistics will however be needed in order to determine the chiral counterterm $\mathscr{N}_{E}^{(2)}$.

\section{References}

[1] L. Cappiello, O. Catà, G. D’ Ambrosio and D. N. Gao, Eur. Phys. J. C 72, 1872 (2012) Erratum: [Eur. Phys. J. C 72, 2208 (2012)] [arXiv:1112.5184 [hep-ph]].

[2] J. R. Batley et al. [NA48/2 Collaboration], Phys. Lett. B 677, 246 (2009) [arXiv:0903.3130 [hep-ex]]; J. R. Batley et al. [NA48/2 Collaboration], Phys. Lett. B 697, 107 (2011) [arXiv:1011.4817 [hep-ex]]; J. R. Batley et al. [NA48/1 Collaboration], Phys. Lett. B 576, 43 (2003) [hep-ex/0309075];

J. R. Batley et al. [NA48/1 Collaboration], Phys. Lett. B 599, 197 (2004) [hep-ex/0409011];

J. R. Batley et al. [NA48/2 Collaboration], Eur. Phys. J. C 68, 75 (2010) [arXiv:1004.0494 [hep-ex]];

C. Lazzeroni et al. [NA62 Collaboration], Phys. Lett. B 732, 65 (2014) [arXiv:1402.4334 [hep-ex]].

[3] G. Ecker, A. Pich and E. de Rafael, Nucl. Phys. B 291, 692 (1987); G. D’Ambrosio, G. Ecker,

G. Isidori and J. Portoles, JHEP 9808, 004 (1998) [hep-ph/9808289]; L. Cappiello and

G. D'Ambrosio, Phys. Rev. D 75, 094014 (2007) [hep-ph/0702292]; G. D'Ambrosio and J. Portoles, Phys. Lett. B 386, 403 (1996) Erratum: [Phys. Lett. B 389, 770 (1996)] Erratum: [Phys. Lett. B 395, 389 (1997)] [hep-ph/9606213].

[4] B. Bloch-Devaux [NA48/2 Collaboration], J. Phys. Conf. Ser. 800, no. 1, 012029 (2017).

[5] C. Marin Benito [LHCb Collaboration], J. Phys. Conf. Ser. 800, no. 1, 012031 (2017). 
[6] G. D’Ambrosio, D. Greynat and G. Vulvert, Eur. Phys. J. C 73, no. 12, 2678 (2013) [arXiv:1309.5736 [hep-ph]]; G. Colangelo, R. Stucki and L. C. Tunstall, Eur. Phys. J. C 76, no. 11, 604 (2016) [arXiv:1609.03574 [hep-ph]].

[7] H. Pichl, Eur. Phys. J. C 20, 371 (2001) [hep-ph/0010284].

[8] L. Cappiello, O. Catà and G. D’Ambrosio, Eur. Phys. J. C 78, no. 3, 265 (2018) [arXiv:1712.10270 [hep-ph]].

[9] A. Lai et al. [NA48 Collaboration], Phys. Lett. B 496, 137 (2000).

[10] A. Lai et al. [NA48 Collaboration], Eur. Phys. J. C 30, 33 (2003).

[11] J. K. Elwood, M. B. Wise and M. J. Savage, Phys. Rev. D 52, 5095 (1995) Erratum: [Phys. Rev. D 53, 2855 (1996)] [hep-ph/9504288].

[12] A. Pich and E. de Rafael, Nucl. Phys. B 358, 311 (1991); G. Ecker, A. Pich and E. de Rafael, Phys. Lett. B 237, 481 (1990); G. D’ Ambrosio and J. Portoles, Nucl. Phys. B 533, 494 (1998) [hep-ph/9711211]; L. Cappiello, O. Catà and G. D’Ambrosio, Phys. Rev. D 85, 015003 (2012) [arXiv:1106.0467 [hep-ph]].

[13] J. R. Batley et al. [NA48/2 Collaboration], arXiv:1809.02873 [hep-ex]. 